\title{
Analysis of an annular PZT actuator for a droplet ejector
}

\author{
D.-A. Wang ${ }^{\mathrm{a},{ }^{*}}$, C.-H. Cheng ${ }^{\mathrm{b}}$, Y.-H. Hsieh ${ }^{\mathrm{a}}$, Z.-X. Zhang ${ }^{\mathrm{c}}$ \\ ${ }^{a}$ Institute of Precision Engineering, National Chung Hsing University, Taichung 402, \\ Taiwan, $R O C$ \\ ${ }^{b}$ Mechanical \& Automation Engineering, Da-Yeh University, Chang-Hua 515, Taiwan, \\ $R O C$ \\ ${ }^{c}$ Au Optronics, Hsinchu, Taiwan, ROC
}

March 22, 2007

\begin{abstract}
An analytical solution for deflection of an annular lead zirconate titanate (PZT) actuator with application in droplet ejection is developed. A linear strain distribution across the thickness direction is assumed in the derivation of the analytical solution. The analytical results agree well with finite element analyses. Fabrication process of a droplet ejector actuated by the PZT is described. The PZT is prepared by powder metallurgy and patterned with poling electrodes. The ejector is fabricated by nickel electroforming. Experimental results of static deflection of the fabricated device confirm the effectiveness of the analytical solution. Droplet ejection of the ejector is demonstrated.
\end{abstract}

Keywords: PZT actuator; Analytical analysis; Droplet ejector; Electroforming

\footnotetext{
${ }^{*}$ Corresponding author. Tel.:+886-4-22857207; fax:+886-4-22858362

E-mail address: daw@dragon.nchu.edu.tw (D.-A. Wang).
} 


\section{Introduction}

Use of improved piezoelectric actuators in the development of micropumps, droplet ejectors, ultrasonic sensors for various applications continues to be a field of interest. PZT ceramic actuators of annular or circular shapes have been proposed and investigated by many researchers, for example, see [1-5]. In comparing with the structure of an annular PZT and a circular PZT, the annular PZT offers higher electromechanical coupling in the radial direction [6]. The high electromechanical coupling implies that an annular structure is more efficient in converting mechanical energy to electrical energy, and vice versa, which is essential for a high performance PZT [7]. Therefore, annular PZTs are suitable for low-cost and low-voltage applications.

Finite element method (FEM) has been used to study and analyze behaviors of annular or circular PZTs [7-9]. Using FEM, detailed stress and strain distributions can be obtained. However, typical FEM can not provide insight of how the behaviors vary with geometry and material properties. To predict and optimize performance of the annular or circular PZTs, many analytical models have been developed. Dobrucki and Pruchnicki [10] gave a deflection solution for a circular PZT bimorph. A linear strain distribution across the thickness direction is assumed in their work. Li and Chen [11] derived an analytical equation for a circular PZT bonded on a circular plate. By assuming a residual stress in the plate, their analytical solution agrees with experimental results. Tzou and Zhou [12] obtained a deflection solution for a circular plate sandwiched between two thin circular PZTs with an initial non-linear large deformation in order to control the plate's deflection and natural frequency. 
Based on available plate solutions and considering forces and moments at the interface between the plate and the PZT, Fox et al. [13] presented analytical solutions for deflections of a circular plate with an annular PZT. Percin et al. [14] applied Kirchhoff plate theory and Mindlin plate theory [15] to derive deflection solutions for thin and thick circular plates with annular PZTs, respectively. A two-port equivalent circuit network is used for numerical calculation of their solutions.

In this paper, we develop a new analytical solution for static deflection of an annular PZT actuator for a droplet ejector. The derivation is based on a procedure described by Li and Chen [11] for their circular PZT actuator. Finite element analyses based on an axisymmetric model for the actuator are carried out to verify the analytical solutions. Fabrication of a droplet ejector with a nickel electroforming process and a powder metallurgy technique is described. An experimental setup and visualization results of the droplet ejector are reported. The experimental results are compared with the analytical solutions and the results of finite element analyses.

\section{Actuator analysis}

An annular PZT actuator, as shown in Fig. 1, is developed to drive droplet ejectors. The actuator is made by bonding an annular PZT element to one side of a passive plate. When an electric field is applied across the thickness, the PZT element strains transversely and radially. The radial strain causes the surface of the passive plate to expand or contract, which causes the entire structure to bend. The active bending can be employed to eject droplets. Fig. 2 schematically shows a concept of a droplet ejector based on the principle. 
For convenience of analysis, we divide the PZT actuator into two parts: one is the three-layer structure including the annular PZT, the bonding material, and the passive plate, the other is the rest of the passive plate part. The moment balance for each part is shown in Fig. 3. A cylindrical coordinate system is also shown in the figure. The analysis below follows the basic procedure described by $\mathrm{Li}$ and Chen [11] for their circular PZT actuator.

Since the three-layer structure is thin compared to its radius of curvature, a linear strain distribution across the thickness direction can be assumed [11]. The $z$ coordinate $-h_{n}$ of the neutral surface is found to be $[10,11]$

$$
-h_{n}=-\frac{\frac{E_{p} h_{p}^{2}}{1-v_{p}^{2}}+\frac{E_{b}\left[\left(h_{b}+h_{p}\right)^{2}-h_{p}^{2}\right]}{1-v_{b}^{2}}+\frac{E_{p z t}\left[\left(h_{b}+h_{p}+h_{p z t}\right)^{2}-\left(h_{b}+h_{p}\right)^{2}\right]}{1-v_{p z t}^{2}}}{\frac{E_{p} h_{p}}{1-v_{p}^{2}}+\frac{E_{p} h_{b}}{1-v_{b}^{2}}+\frac{E_{p} h_{p z t}}{1-v_{p z t}^{2}}}
$$

where $E, v$ and $h$ are the elastic modulus, Poisson's ratio and thickness, respectively. The subscript $p, b$ and pzt represent the passive plate, bonding layer and PZT, respectively. The radial strain $\varepsilon$ located a distance $z$ from the neutral surface can be expressed as

$$
\varepsilon=z \kappa
$$

where $\kappa$ is the curvature of the neutral surface. The PZT material can be assumed to be elastically isotropic when the working frequency of the droplet ejector is much lower than the resonant frequency of the PZT [11].

Assuming that the radial and circumferential strains are equal in the structure and using Eq. (2), the radial stress $\sigma_{b}$ of the bonding material under plane stress condition is 


$$
\sigma_{b}=\frac{E_{b}}{1-v_{b}} \varepsilon_{b}=\frac{E_{b}}{1-v_{b}} z \kappa
$$

where $\varepsilon_{b}$ is the radial strain of the bonding layer. Similarly, the radial stress $\sigma_{i}$ of the interface between the bonding layer and passive plate, and the radial stress $\sigma_{p}$ of the passive plate can be written as

$$
\begin{aligned}
& \sigma_{i}=\frac{E_{b}}{1-v_{b}}\left(h_{p}-h_{n}\right) \kappa=\frac{E_{p}}{1-v_{p}}\left(h_{p}-h_{n}\right) \kappa \\
& \sigma_{p}=\frac{E_{p}}{1-v_{p}} z \kappa=\frac{\sigma_{i} z}{h_{p}-h_{n}}
\end{aligned}
$$

Combining Eqs. (3), (4) and (5), we have

$$
\sigma_{b}=\frac{E_{b}\left(1-v_{p}\right)}{E_{p}\left(1-v_{b}\right)} \frac{\sigma_{i}}{h_{p}-h_{n}} z
$$

The constitutive equation of the PZT is given as

$$
\sigma_{p z t}=\frac{E_{p z t}}{1-v_{p z t}}\left(z \kappa-\frac{V}{h_{p z t}} d_{31}\right)
$$

where $V$ is the applied voltage and $d_{31}$ is the piezoelectric strain constant. Substituting Eq. (4) into Eq. (7), we have

$$
\sigma_{p z t}=\frac{E_{p z t}}{\left(1-v_{p z t}\right)}\left(\frac{\sigma_{i}\left(1-v_{p}\right)}{E_{p}\left(h_{p}-h_{n}\right)} z-\frac{V}{h_{p z t}} d_{31}\right)
$$

Balancing the moments in the three-layer structure, an expression for $\sigma_{i}$ is obtained as

$$
\sigma_{i}=\frac{3 \eta V d_{31} h^{\prime}\left(2 h^{\prime}+2 h_{b}+h_{p z t}\right)}{2\left[\left(3 h^{\prime 2}+3 h^{\prime} h_{b}+h_{b}^{2}\right) h_{b} \lambda+\left(\left(h^{\prime}+h_{b}+h_{p z t}\right)^{3}-\left(h^{\prime}+h_{b}\right)^{3}\right) s+h^{\prime 3}+h_{n}^{3}\right]}
$$


where $h^{\prime}=h_{p}-h_{n}, \eta=\frac{E_{p z t}}{1-v_{p z t}}, \lambda=\frac{E_{b}\left(1-v_{p}\right)}{E_{p}\left(1-v_{b}\right)}$ and $\varsigma=\frac{E_{p z t}\left(1-v_{p}\right)}{E_{p}\left(1-v_{p z t}\right)}$. The bending moments per unit length $M_{1}$ and $M_{2}$ are given as

$$
\begin{aligned}
M_{1}=M_{2} & =\int_{-h_{n}}^{h^{\prime}} \sigma_{p} z d z \\
& =\frac{\eta V d_{31}\left(2 h^{\prime}+2 h_{b}+h_{p z t}\right)\left(h^{\prime 3}+h_{n}^{3}\right)}{2\left[\left(3 h^{\prime 2}+3 h^{\prime} h_{b}+h_{b}^{2}\right) h_{b} \lambda+\left(\left(h^{\prime}+h_{b}+h_{p z t}\right)^{3}-\left(h^{\prime}+h_{b}\right)^{3}\right) s+h^{\prime 3}+h_{n}^{3}\right]}
\end{aligned}
$$

Assuming the passive plate is simply supported at its outer edge, i.e. $M_{3}=0$, the deflection $w_{1}$ of the passive plate for $a<r \leq c$ is

$$
w_{1}(r)=\frac{-b^{2} M_{22}\left(c^{2}-r^{2}\right)}{2 D_{p}\left(1+v_{p}\right)\left(c^{2}-a^{2}\right)}+\frac{a^{2} b^{2} M_{22}}{D_{p}\left(1-v_{p}\right)\left(c^{2}-a^{2}\right)} \ln \frac{r}{c}
$$

where $D_{p}=\frac{E_{p} h_{p}^{3}}{12\left(1+v_{p}^{2}\right)} \cdot c$ is the radius of the plate, and $a$ is the outer radius of the annular PZT. The deflection $w_{2}$ of the three-layer structure for $b<r \leq a$ is

$$
w_{2}(r)=\frac{\left(a^{2} M_{2}-b^{2} M_{1}\right)\left(r^{2}-a^{2}\right)}{2 D_{c}\left(1+v_{c}\right)\left(a^{2}-b^{2}\right)}-\frac{b^{2} M_{22}}{2 D_{p}\left(1+v_{p}\right)}+\frac{a^{2} b^{2} M_{22}}{D_{p}\left(1-v_{p}\right)\left(c^{2}-a^{2}\right)} \ln \frac{a}{c}
$$

where $D_{c}=\frac{E_{c}\left(h_{p z t}+h_{b}+h_{p}\right)^{3}}{12\left(1+v_{c}^{2}\right)} . \quad E_{c}$ and $v_{c}$ are the elastic modulus and Poisson's ratio for the three-layer structure, respectively. Based on Christensen's work [16], $E_{c}$ and $v_{c}$ are derived as

$$
\begin{aligned}
& E_{c}=f_{1} E^{\prime}+f_{2} E_{p}+\frac{f_{1} f_{2} E^{\prime} E_{p}\left(v_{p}-v^{\prime}\right)^{2}}{f_{1} E_{p}\left(1-v^{\prime 2}\right)+f_{2} E^{\prime}\left(1-v_{p}^{2}\right)} \\
& v_{c}=\frac{f_{1} E_{p} v_{p}\left(1-v^{\prime 2}\right)+f_{2} E^{\prime} v^{\prime}\left(1-v_{p}^{2}\right)}{f_{1} E_{p}\left(1-v^{\prime 2}\right)+f_{2} E^{\prime}\left(1-v_{p}^{2}\right)}
\end{aligned}
$$


where $f_{1}=\frac{h_{p}}{h_{p z t}+h_{b}+h_{p}}$ and $f_{2}=\frac{h_{p z t}+h_{b}}{h_{p z t}+h_{b}+h_{p}} . E^{\prime}$ and $v^{\prime}$ are given as

$$
\begin{aligned}
& E^{\prime}=f_{3} E_{p z t}+f_{4} E_{b}+\frac{f_{3} f_{4} E_{p z t} E_{b}\left(v_{p z t}-v_{b}\right)^{2}}{f_{3} E_{p z t}\left(1-v_{b}^{2}\right)+f_{4} E_{b}\left(1-v_{p z t}^{2}\right)} \\
& v^{\prime}=\frac{f_{3} E_{p z z} v_{p z t}\left(1-v_{b}^{2}\right)+f_{4} E_{b} v_{b}\left(1-v_{p z t}^{2}\right)}{f_{3} E_{p z t}\left(1-v_{b}^{2}\right)+f_{4} E_{b}\left(1-v_{p z t}^{2}\right)}
\end{aligned}
$$

where $f_{3}=\frac{h_{p z t}}{h_{p z t}+h_{b}}$ and $f_{4}=\frac{h_{b}}{h_{p z t}+h_{b}}$. The deflection $w_{3}$ of the passive plate for $0 \leq r \leq b$ is

$$
\begin{gathered}
w_{3}(r)=\frac{M_{11}\left(b^{2}-r^{2}\right)}{2\left(1+v_{p}\right) D_{p}}+\frac{\left(b^{2} M_{1}-a^{2} M_{2}\right)}{2\left(1+v_{c}\right) D_{c}}-\frac{b^{2} M_{22}}{2\left(1+v_{p}\right) D_{p}} \\
+\frac{a^{2} b^{2} M_{22}}{\left(1-v_{p}\right) D_{p}\left(c^{2}-a^{2}\right)} \ln \frac{a}{c}
\end{gathered}
$$

The moments $M_{22}$ and $M_{11}$ are determined by continuity conditions $\left.\frac{d w_{1}}{d r}\right|_{r=a}=\left.\frac{d w_{2}}{d r}\right|_{r=a}$ and $\left.\frac{d w_{2}}{d r}\right|_{r=b}=\left.\frac{d w_{3}}{d r}\right|_{r=b}$, respectively, as

$$
\begin{aligned}
& M_{22}=\left[\frac{2 a C_{1}}{C_{2}+C_{3}}\right]^{1 / 2} \\
& M_{11}=-2 D_{p}\left(1+v_{p}\right) C_{1}
\end{aligned}
$$

where $C_{1}=\frac{2\left(a^{2} M_{2}-b^{2} M_{1}\right)}{D_{c}\left(1+v_{c}\right)\left(a^{2}-b^{2}\right)}, C_{2}=\frac{a b^{2}}{D_{p}\left(1+v_{p}\right)\left(c^{2}-a^{2}\right)}$ and $C_{3}=\frac{a b^{2}}{D_{p}\left(1-v_{p}\right)\left(c^{2}-a^{2}\right)}$. 


\section{Finite element analyses}

In order to obtain accurate static deflection solutions for the ejector and to examine the validity of the analytical solutions of Eq. (17), finite element analyses are carried out. Due to symmetry, an axisymmetric model of the ejector is considered. Fig. 4(a) shows a schematic of an axisymmetric slice of the ejector. The ejector has the thicknesses $h_{p z t}(=300 \mu \mathrm{m}), h_{b}(=5 \mu \mathrm{m}), h_{p}(=80 \mu \mathrm{m})$ and the radiuses $c(=8 \mathrm{~mm})$, $a(=6 \mathrm{~mm}), b(=4 \mathrm{~mm})$ according to the fabricated devices. A cylindrical coordinate system is also shown in the figure. As shown in Fig. 4(a), the displacements in the $r$ and $z$ directions at the bottom of the outer edge surface of the passive plate are constrained to represent the assumed simply supported boundary conditions. The displacement in the $r$ direction of the symmetry axis, the $z$ axis, is constrained. The actuating voltage $V$ is applied at the top surface of the PZT. Fig. 4(b) shows a mesh for a finite element model. Fig. 4(c) shows a close-up view of the mesh near the inner edge of the PZT. The finite element model has 1,840 8-node quadratic elements.

In this investigation, the PZT, bonding layer and passive plate are assumed to be linear elastic isotropic materials. The material properties of the PZT, bonding layer and passive plate are listed in Table 1. The commercial finite element program ABAQUS [17] is employed to perform the computations. 8-node quadratic element CAX8R is used to model the bonding layer and passive plate. Piezoelectric element CAX8RE is used to model the PZT.

The analytical solution of Eq. (17) is compared to the results of FEM. As shown in Fig. 5, the analytical solutions of the deflection at the center of the passive plate agree well with the FEM results. For the DC actuating voltage ranging from $20 \mathrm{~V}$ to $100 \mathrm{~V}$, the 
largest discrepancy between the analytical solutions and FEM results is only $1.6 \%$. This error might be due to the assumption of equal radial and circumferential strains in the structure. Based on three-dimensional finite element analyses of the PZT actuator, this assumption is not valid near the inner and outer edges of the PZT and the edge of the passive plate.

\section{Fabrication, experiments and discussions}

In order to verify the effectiveness of the analytical solution of Eq. (17), a prototype of a droplet ejector actuated by an annular PZT actuator is fabricated. In the experiments, we emphasize on the static analysis of the PZT actuator. The experiments presented in this investigation are mainly for verification of the developed analytical solution of the static deflection of the PZT actuator. The analytical solution is not used in the design of the fabricated actuator.

\subsection{Fabrication}

The passive plate with a nozzle at its center is fabricated by nickel electroforming process on a stainless steel substrate. Fig. 6 shows the fabrication steps. First, a $4 \mu \mathrm{m}$ thick photoresist mould (AZ9260) is coated and patterned over the substrate. Next, a 60 $\mu \mathrm{m}$-thick nickel layer is electrodeposited using a low-stress nickel sulfamate bath with the chemical compositions listed in Table 2. The bath is kept at a temperature of $60{ }^{\circ} \mathrm{C}$ and a $\mathrm{pH}$ value around 4.0. The current density is $2.7 \mathrm{~A} / \mathrm{dm}^{2}$. A nozzle is formed by over-deposition of nickel around the columnar photoresist mould. Then, the nickel 
microstructure is removed from the substrate. Fig. 7 shows a photo and a SEM of an electroformed nozzle. The diameter of the nozzle is $38 \mu \mathrm{m}$.

The annular PZT is prepared by poling the PZT powder compact that has been sintered and polished. Detail about the fabrication process is described by Cheng et al. [18]. Subsequently, The PZT is glued to the passive plate by an epoxy adhesive (3M, DP-460) cured at a temperature of $60^{\circ} \mathrm{C}$. Finally, the assembly is glued to a liquid chamber made of acrylic, as shown in Fig. 8.

\subsection{Experiments}

The deflection of the passive plate of the droplet ejector operating in air is measured by a Polytec MSV300 laser Doppler vibrometer. DC voltages are applied to the PZT actuator. The experimental results, as shown in Fig. 5, are slightly lower than the analytical solution of Eq. (17). Li and Chen [11] pointed out that bonding process often introduce residual stress in the passive plate. FEM computations with a $0.8 \mathrm{MPa}$ residual stress are carried out. As shown in Fig. 5, the results of the FEM with a $0.8 \mathrm{MPa}$ residual stress agree well with the experimental results.

The residual stress in the passive plate can be attributed to the bonding process and/or the electroforming process. Hadian and Gabe [19] reported that the residual $\underline{\text { stresses in the electrodeposits of nickel under the electroplating parameters of the current }}$ density $\left(=2.8 \mathrm{~A} / \mathrm{dm}^{2}\right)$ and the $\mathrm{pH}(=3.75-4)$ in the Watts bath are $0.6 \pm 0.6 \mathrm{MPa}$. These parameters are close to the current density, $2.7 \mathrm{~A} / \mathrm{dm}^{2}$, and the $\mathrm{pH}$, around 4 , used in our experiments. This may justify the use of the $0.8 \mathrm{MPa}$ residual stress in the simulation. However, the nickel microstructures removed from the substrates after the 
electroforming process are nearly flat. This indicates that their residual stresses are small and may be neglected. The epoxy adhesive for gluing the PZT to the passive plate is cured at a temperature of $60{ }^{\circ} \mathrm{C}$. Due to the difference in the thermal expansion coefficients of the materials, the curing process may be the main source of the $0.8 \mathrm{MPa}$ residual stress found from the simulation.

From the mechanics viewpoint, the tensile residual stress inside the plate may bend the assembly upward. Note that the passive plate is placed under the PZT. Since the DC voltages are applied to deflect the plate downward in the experiments and simulation, the experimental results should be lower than the simulation results without consideration of residual stress. Higher curing temperatures may introduce higher residual stresses, and the performance of the PZT can be further deteriorated.

To demonstrate the ejector's operation and to characterize the droplet ejection sequence, a visualization system, shown in Fig. 9, is developed. A LED is placed under the droplet ejector to side-illuminate the droplet stream. The ejector is arranged to eject droplets vertically under influence of gravity. Two signals, synchronized with adjustable time delay, are sent to the ejector and the LED, respectively. Droplets are ejected from the ejector by a voltage pulse train at $7.3 \mathrm{kHz}$ and $\mathrm{V}_{\mathrm{pp}}$ of $80 \mathrm{~V}$. The droplet images are frozen by flashlight from the LED at specified time delays and acquired by a CCD camera. Fig. 10 shows a droplet ejection sequence of the ejector. Upto $60 \mu$ s after ejection, the primary droplet is shown to be dragging a long tail, which subsequently breaks into several smaller droplets by the action of capillary instability [20]. The droplet behaviour is connected to the action of the PZT. As described by Persin et al. [3], the passive plate is set into vibration when the voltage pulse train is applied to the PZT. The 
fluid behind the orifice is accelerated as the passive plate moves. When the inertial force is larger than the surface tension force that hold it to the orifice, a drop is ejected from the orifice.

\subsection{Effects of PZT types}

$\underline{\text { In this investigation, only one type of the PZT materials, PZT-5A, is employed for }}$ the verification of the analytical solution and the experiments. It is worthwhile to have a comparison of the static performance of different types of PZT. Three PZT materials are considered, namely PZT-5A, PZT-5H, and PZT-4. The material properties of PZT-5H and PZT-4 are listed in Table 3. Fig. 11 shows the analytical solutions of the deflection at the center of the passive plate actuated by the PZTs. As the DC actuating voltage increases, the deflections increase monotonically. For the DC actuating voltages ranging from $0 \mathrm{~V}$ to $100 \mathrm{~V}$, the deflections of the plate actuated by the PZT (PZT-4) are much smaller than those actuated by the other two types of the PZTs. Among the three PZTs, the deflection of the plate actuated by the PZT (PZT-5H) is the largest. This is due to its largest value of the piezoelectric strain constant $e_{31}$ among the PZTs. Compared to the PZT-5A, the PZT-5H has higher sensitivity and permittivity. However, its markedly lower Curie point limits the working temperature range and leads to lower temperature stability [21]. The high resistivity at elevated temperatures, high sensitivity and high time stability of PZT-5A make it a better candidate for instrument applications. 


\section{Conclusions}

An analytical solution for analysis of static deflection of an annular PZT actuator is derived. The analytical solution is verified by finite element analyses and experimental results. This solution can be used to predict the static performance of the PZT actuator with different dimensions and material properties. A droplet ejector actuated by the PZT has been fabricated and tested to verify the developed analytical solution of the static deflection of the PZT actuator. The droplet ejector can be used as a cost-effective miniaturized module for ink jet printers.

\section{Acknowledgement}

The authors would like to express their appreciation to the National Center for High-Performance Computing (NCHC), Taiwan for their assistance. Helpful discussions with Mr. Yu-Ching Shih of NCHC are greatly appreciated. 


\section{References}

[1] E. Stemme and G. Stemme, A valveless diffuser/nozzle-based fluid pump, Sensors and Actuators A, 39 (1993) 159-167.

[2] A. Olsson, G. Stemme and E. Stemme, A valve-less planar fluid pump with two pump chambers, Sensors and Actuators A, 46-47 (1995) 549-556.

[3] G. Percin, T. S. Lundgren and B. T. Khuri-Yakub, Controlled ink-jet printing and deposition of organic polymers and solid particles, Applied Physics Letters, 73 (1998) 2375-2377.

[4] J.-H. Hu, H.-L. Li, H. Chan and C.-L. Choy, A ring-shaped piezoelectric transformer operating in the third symmetric extensional vibration mode, Sensors and Actuators A, 88 (2001) 79-86.

[5] I. Izzo, D. Accoto, A. Menciassi, L. Schmitt and P. Dario, Modeling and experimental validation of a piezoelectric micropump with novel no-moving-part valves, Sensors and Actuators A, 133 (2007) 128-140.

[6] H.-L. Li, H. L.-W. Chan and C.-L. Choy, Vibration characteristics of piezoceramics rings, Ferroelectrics, 263 (2001) 211-216.

[7] H.-L. Li, J.-H. Hu and H. L.-W. Chan, Finite element analysis on piezoelectric ring transformer, IEEE Transactions on Ultrasonics, Ferroelectrics, and Frequency Control, 51 (2004) 1247-1254.

[8] C. J. Morris and F. K. Forster, Optimization of a circular piezoelectric bimorph for a micropump driver, J. Micromech. Microeng., 10 (2000) 459-465.

[9] S. Jun and Z. Zhaowei, Finite element analysis of a IBM suspension integrated with a PZT microactuator, Sensors and Actuators A, 100 (2002) 257-263.

[10] A. B. Dobrucki and P. Pruchnicki, Theory of piezoelectric axisymmetric bimorph, Sensors and Actuators A, 58 (1997) 203-212.

[11] S. Li and S. Chen, Analytical analysis of a circular PZT actuator for valveless micropumps, Sensors and Actuators A, 104 (2003) 151-161.

[12] H. S. Tzou and Y. H. Zhou, Dynamics and control of non-linear circular plates with piezoelectric actuators, Journal of Sound and Vibration, 188 (1995) 189-207.

[13] C. H. J. Fox, X. Chen, S. McWillian, Analysis of the deflecton of a circular plate with an annular piezoelectric antuator, Sensors and Actuators A, 133 (2007) 180- 
194.

[14] G. Percin, T. Butrus and B. T. Khuri-Yakub, Piezoelectrically actuated flextensional micromachined ultrasound transducers - I: Theory, IEEE Transactions on Ultrasonics, Ferroelectrics, and Frequency Control, 49 (2002) 573-584.

[15] R. D. Mindlin, Influence of rotatory inertia and shear on flexural motions of isotropic, elastic plates, Journal of Applied Mechanics, 73 (1951) 31-38.

[16] R. M. Christensen, Mechanics of composite materials, Wiley, New York, 1979.

[17] H. D. Hibbitt, B. I. Karlsson and E. P. Sorensen, ABAQUS user manual, Version 6-2, 2001

[18] C.-H. Cheng, S.-C. Chen, H.-C. Kuo and Z.-B. Chou, The polling design of a shear mode piezoelectric actuator, Journal of Micromechanics and Microengineering, 15 (2005) 2163-2171.

[19] S. E. Hadian and D. R. Gabe, Residual stresses in electrodeposits of nickel and nickel-iron alloys, Surface and Coatings Technology, 122 (1999) 118-135.

[20] F.-G. Tseng, C.-J. Kim and C.-M. Ho, A high-resolution high-frequency monolithic top-shooting microinjector free of satellite drops - part II: fabrication, implementation, and characterization, Journal of Microelectromechanical Systems, 11 (2002) 437-447.

[21] D. Berlincourt, H. H. A. Krueger and C. Near, Properties of piezoelectricity ceramics, Technical Publication TP-226, Morgan Electro Ceramics, 2007.

\section{Biography}

Dung-An Wang received the Ph.D. degree in mechanical engineering from the University of Michigan at Ann Arbor, in 2004. He is currently an Assistant Professor in the Institute of Precision Engineering, National Chung Hsing University, Taiwan, ROC. His research interests include micromachined resonators and actuators, piezoelectric actuators, microassembly and compliant mechanisms.

Chiang-Ho Cheng received his Ph.D. degree from the Institute of Applied Mechanics in National Taiwan University, Taiwan, ROC, in 1993. He is an Associate Professor in the Mechanical \& Automation Engineering, Da-Yeh University, Taiwan, ROC. His present 
research interests concern piezoelectric actuators and their applications in MEMS, such as microdroplet ejectors, micromirrors, dispensers and micropumps.

Yi-Han Hsieh received the B.S. degree in mechanical engineering from the Southern Taiwan University of Technology, Taiwan, ROC, in 2005, and is currently working towards the M.S. degree in the Institute of Precision Engineering, National Chung Hsing University, Taiwan, ROC. His research interests are micromachined resonators and actuators, and compliant mechanisms.

Zou-Xing Zhang received the M.S. degree in mechanical engineering from the Da-Yeh University, Taiwan, ROC, in 2005. He currently serves in the ROC Army. 
Table 1. Properties of the PZT (PZT-5A), bonding layer (epoxy, 3M DP-460) and passive plate (nickel)

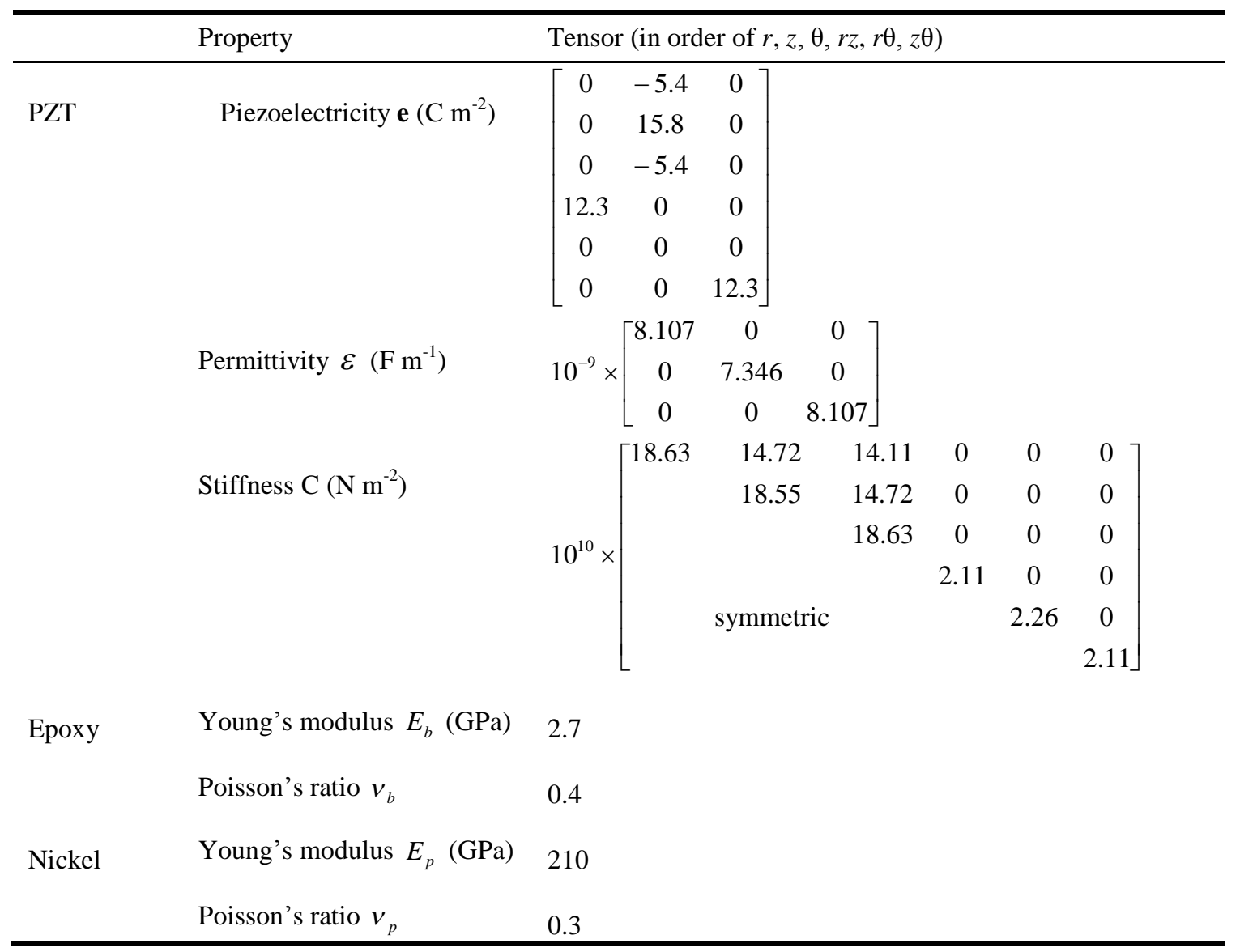

Table 2. Chemical compositions of the low-stress nickel electroplating solution

\begin{tabular}{ll}
\hline Chemical & Amount $(\mathrm{g} / \mathrm{L})$ \\
\hline Nickel sulfamate, $\mathrm{Ni}\left(\mathrm{NH}_{2} \mathrm{SO}_{3}\right)_{2} \cdot 4 \mathrm{H}_{2} \mathrm{O}$ & 500 \\
Nickel chloride, $\mathrm{NiCl}_{2} \cdot 6 \mathrm{H}_{2} \mathrm{O}$ & 5 \\
Boric acid, $\mathrm{H}_{3} \mathrm{BO}_{3}$ & 35 \\
Wetting agent & 5 \\
\hline
\end{tabular}


Table 3. Properties of the PZT-5H and PZT-4.

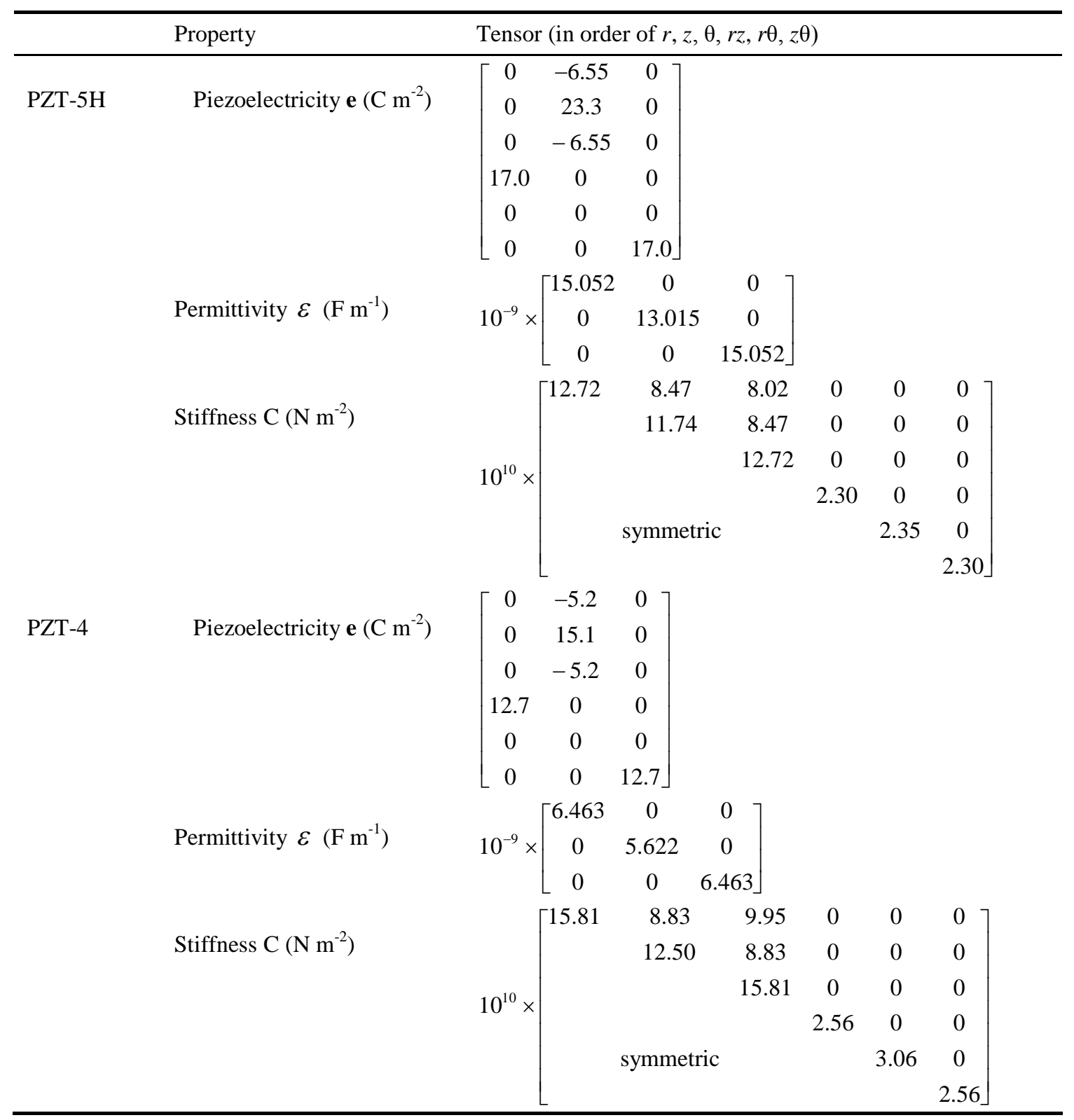




\section{List of figures}

Fig. 1 Schematic of a PZT actuator bonded to a passive plate.

Fig. 2 Schematic of a droplet ejector.

Fig. 3 An ejector loaded by moments.

Fig. 4. (a) Schematic of an axisymmetric slice of the ejector. (b) Finite element model.

(c) Close-up view of the mesh near the inner edge of the PZT.

Fig. 5. Deflection at the center of the passive plate.

Fig. 6. Fabrication steps of a nozzle with nickel electroforming process.

Fig. 7. An electroformed nozzle (a) Photo (b) SEM.

Fig. 8. A prototype of a droplet ejector.

Fig. 9. Visualization system (a) Component diagram (b) Photo.

Fig. 10. Droplet ejection sequence.

Fig. 11. Deflection at the center of the passive plate. 


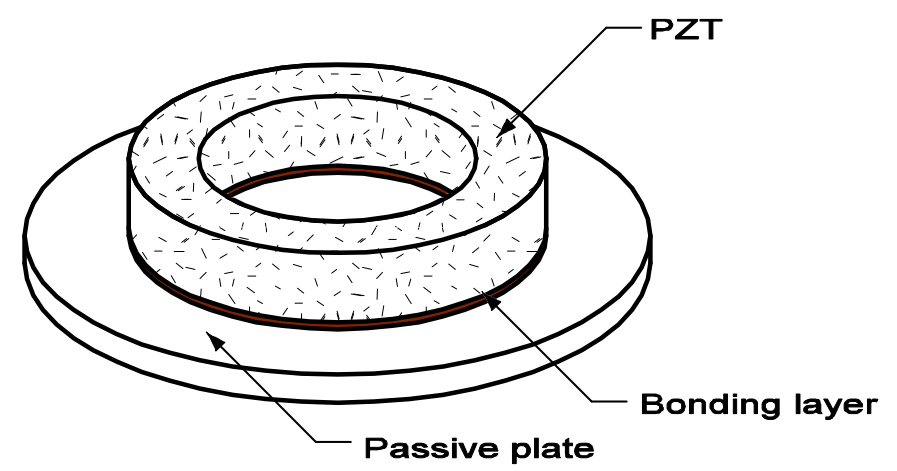

Fig. 1. Schematic of a PZT actuator bonded to a passive plate. 


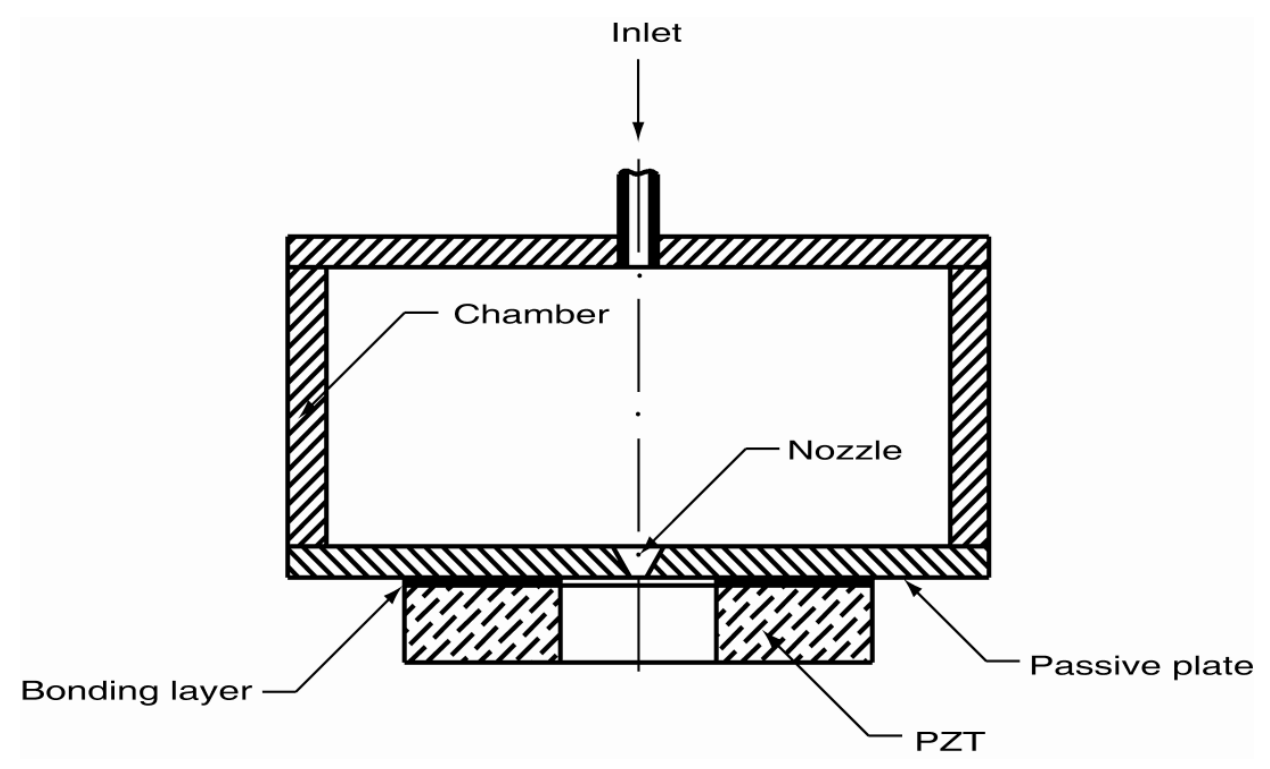

Fig. 2. Schematic of a droplet ejector. 


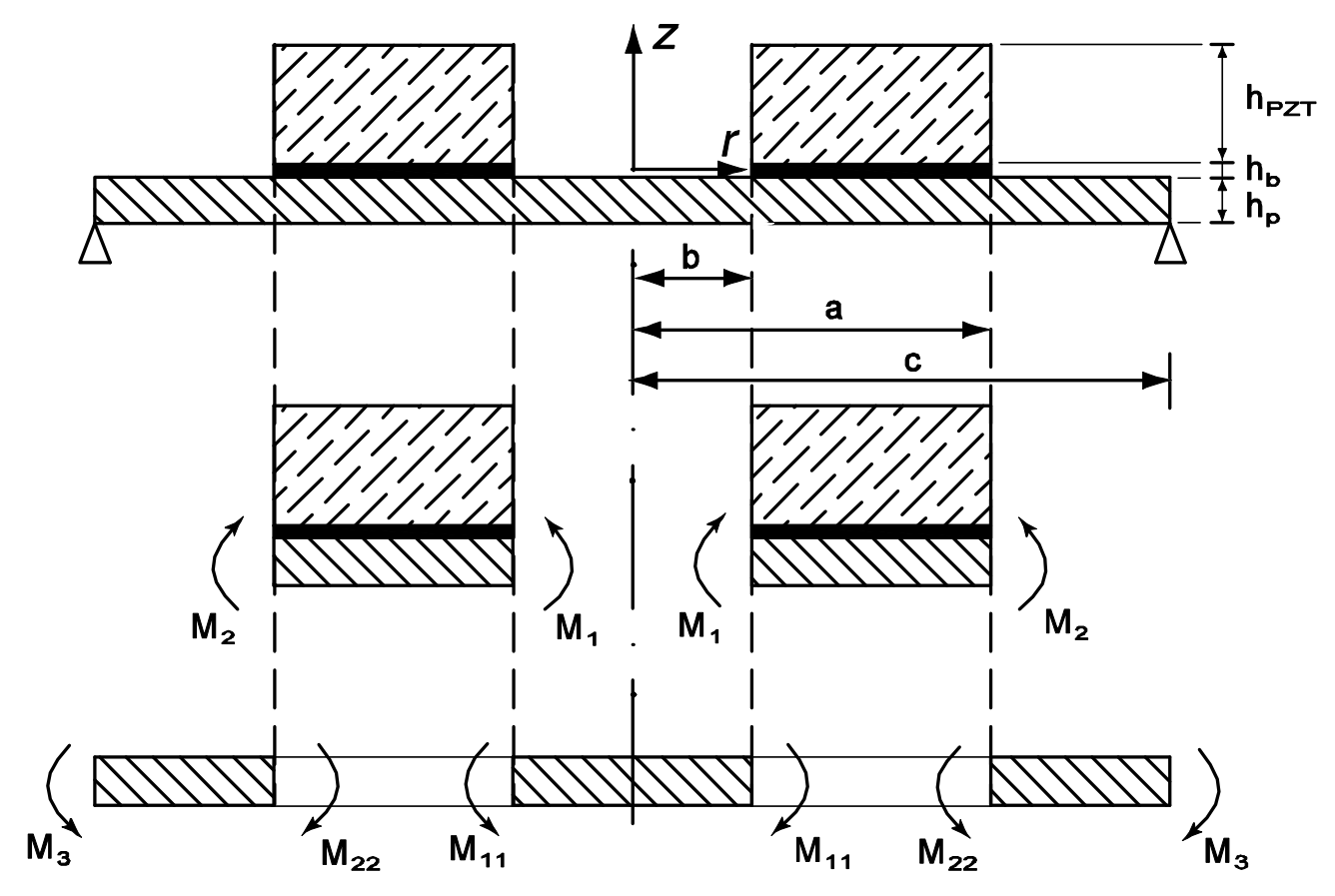

Fig. 3. An ejector loaded by moments. 


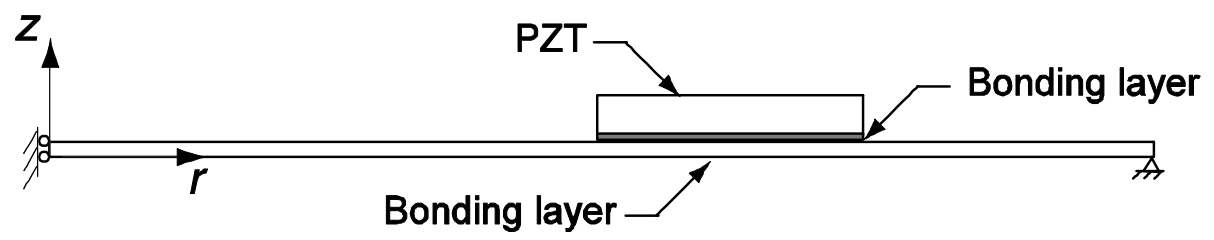

(a)
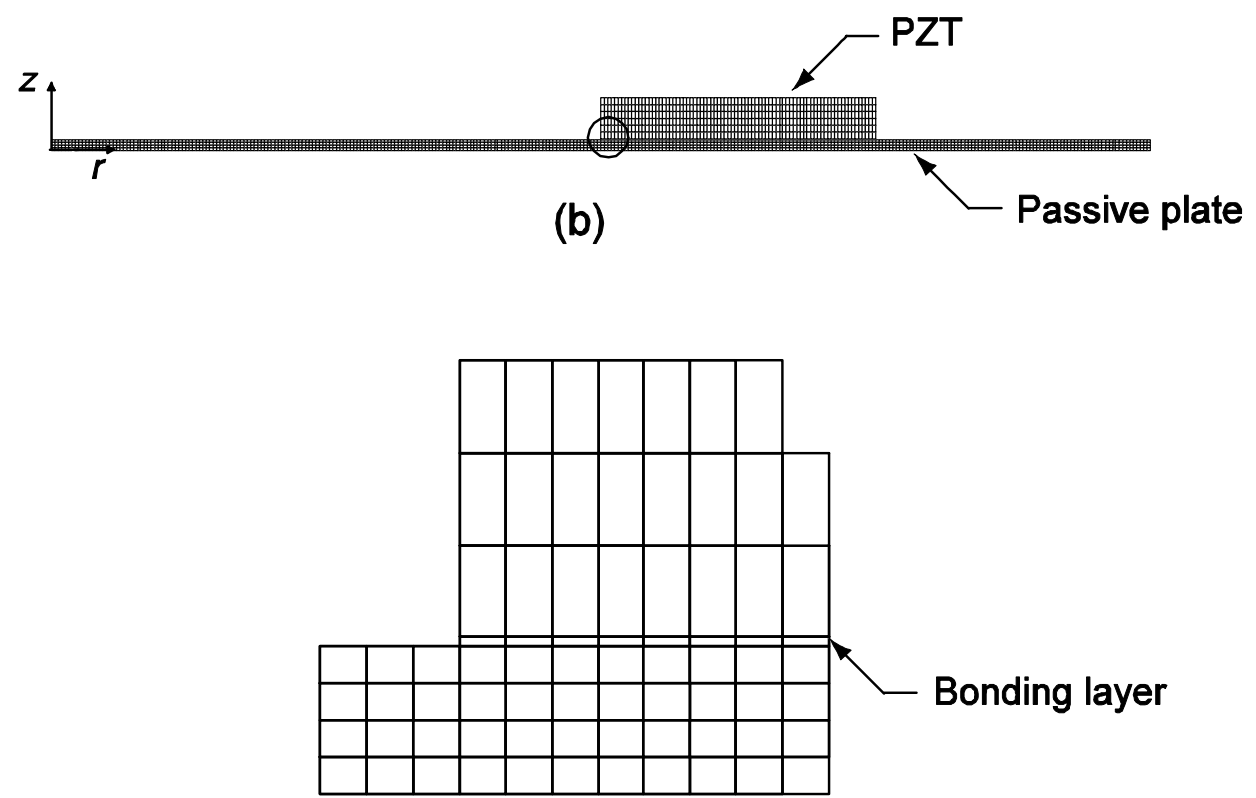

(c)

Fig. 4. (a) Schematic of an axisymmetric slice of the ejector. (b) Finite element model. (c) Close-up view of the mesh near the inner edge of the PZT. 


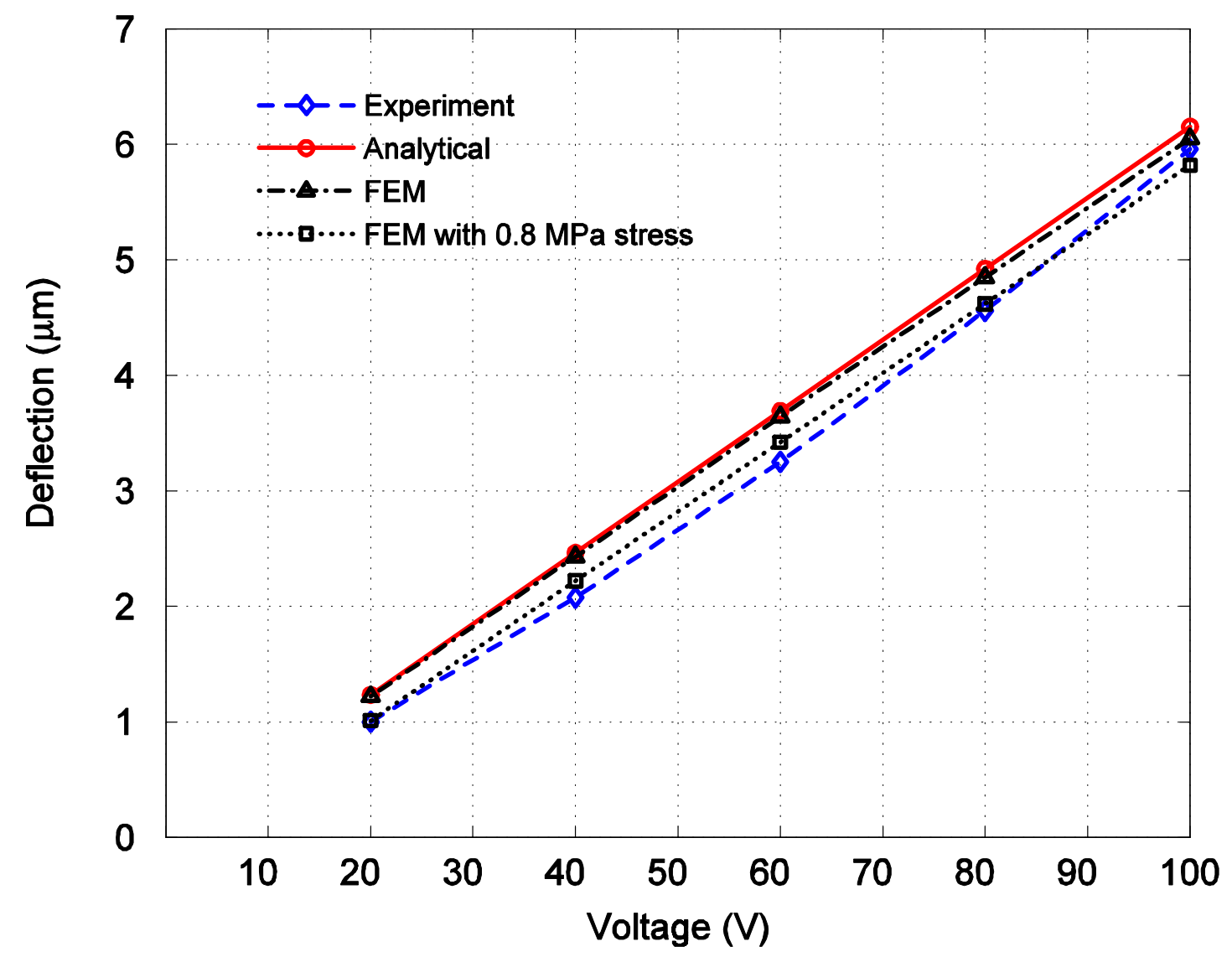

Fig. 5. Deflection at the center of the passive plate. 


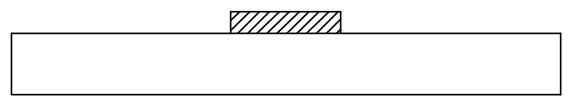

(a) Coat and pattern photoresist

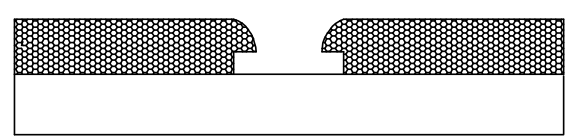

(b) Electroplate $\mathrm{Ni}$

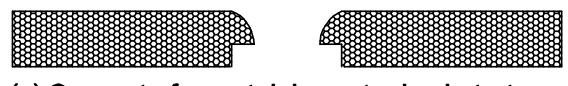

(c) Seperate from stainless steel substrate

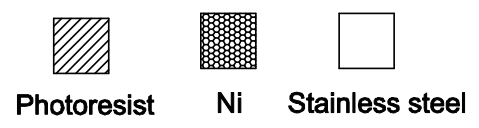

Fig. 6. Fabrication steps of a nozzle with nickel electroforming process. 


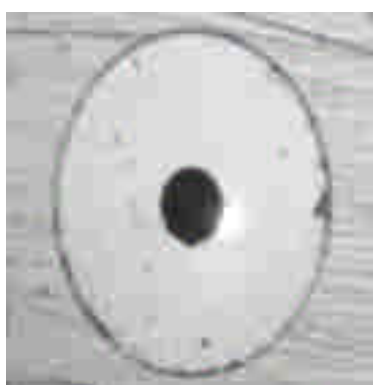

(a)

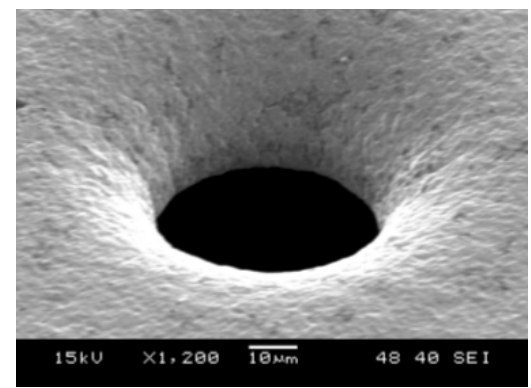

(b)

Fig. 7. An electroformed nozzle (a) Photo (b) SEM. 


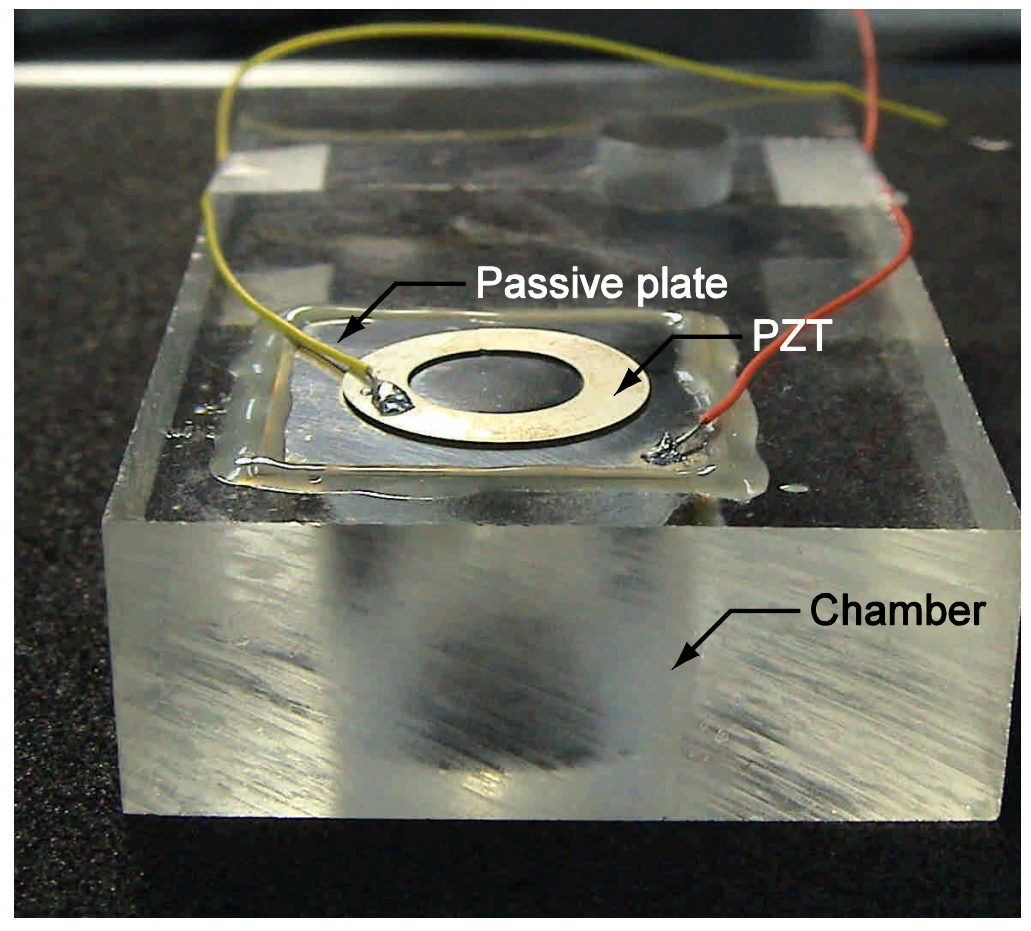

Fig. 8. A prototype of a droplet ejector. 


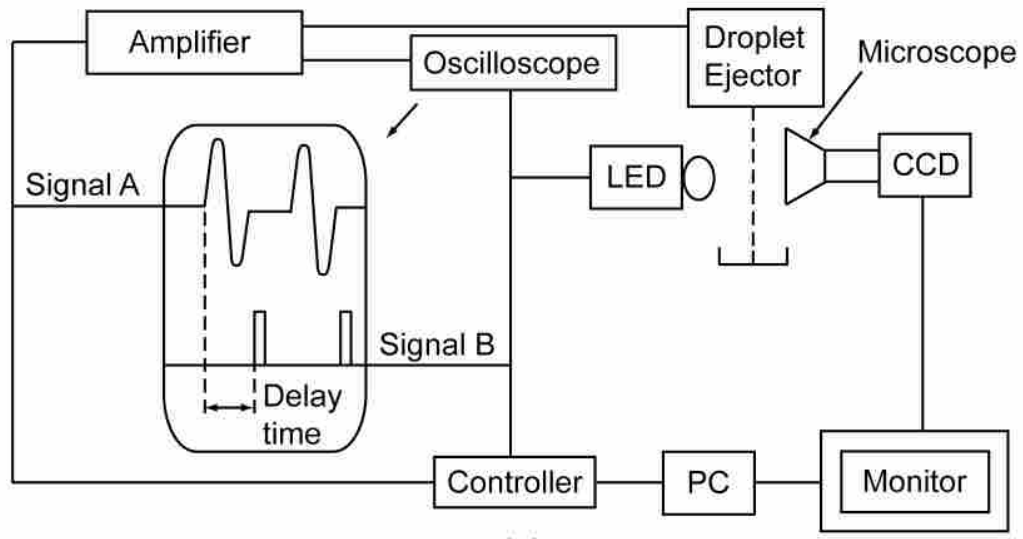

(a)

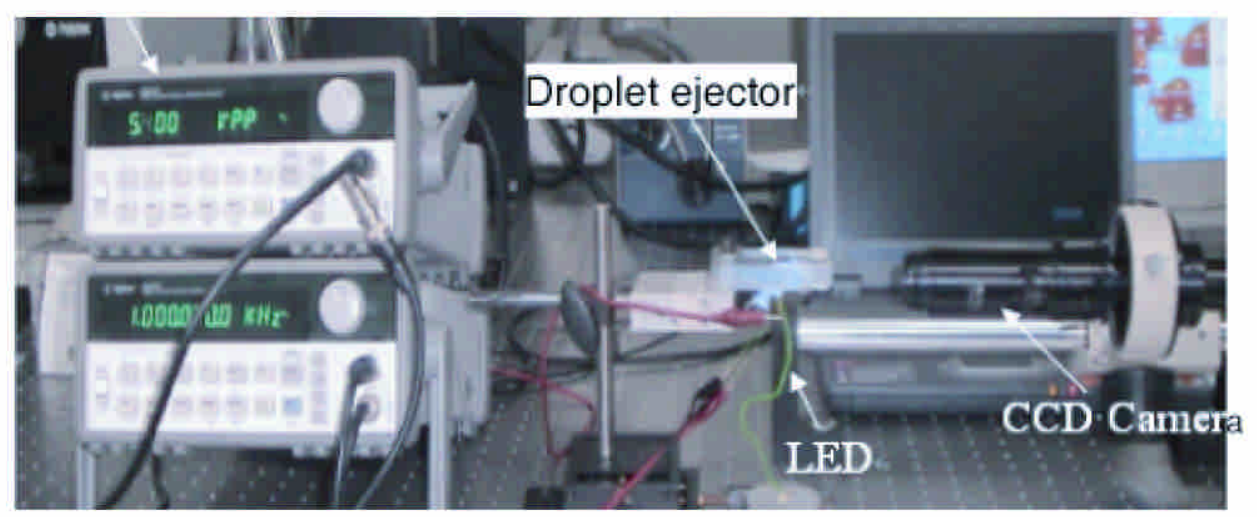

(b)

Fig. 9. Visualization system (a) Component diagram (b) Photo. 


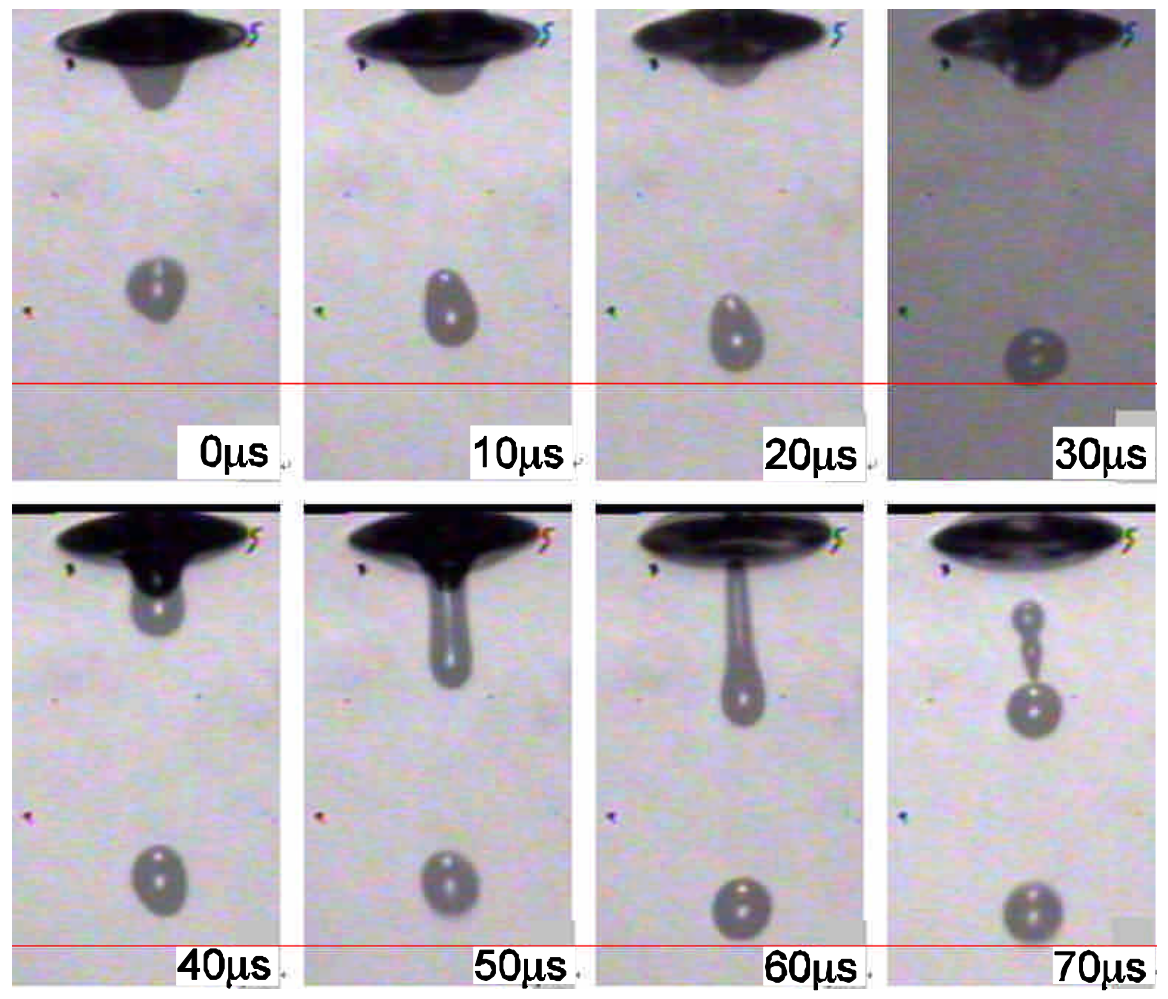

Fig. 10. Droplet ejection sequence. 


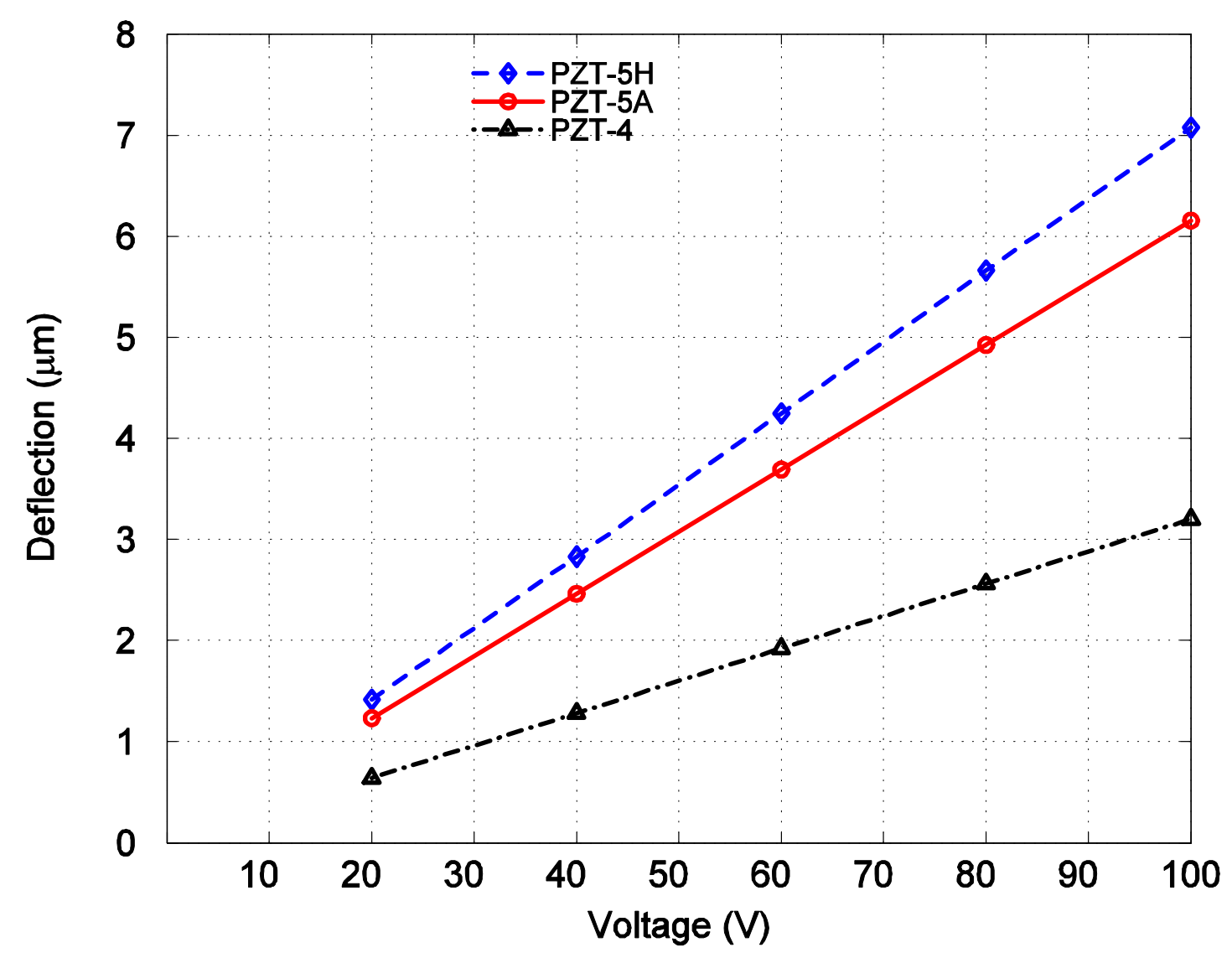

Fig. 11. Deflection at the center of the passive plate. 